[RAdiocarbon, Vol 22, No. 1, 1980, P 82-90]

\title{
MOSCOW MV LOMONOSOV STATE UNIVERSITY RADIOCARBON DATES I
}

\author{
N I GLUSHANKOVA, O B PARUNIN, T A TIMASHKOVA, \\ $\mathrm{V}$ Z KHAIT and A I SHLUKOV
}

Laboratory of Recent Sediments and Pleistocene Paleogeography, Moscow

\section{INTRODUCTION}

The following list sums up the results of radiocarbon age determinations obtained at the Laboratory in 1974-1975. The list is prefaced with a brief description of the measurement procedure and discussion of some methodological aspects of the investigation that result in a higher accuracy of dating.

The radiocarbon dating was performed by the liquid-scintillation method. Benzene synthesized from the samples was used as a measuring preparation. The standard was provided by the Geological Institute of the Academy of Sciences of the USSR of which the conversion factor is 1.1922 relative to $95 \%$ of the activity of NBS oxalic acid.

The age measurements cited below were calculated using the ${ }^{14} \mathrm{C}$ carbon half-life obtained by Libby, $5570 \pm 30$ years. The sample age was measured in radiocarbon years prior to 1950. Correction for isotopic fractionation was not made. Radiocarbon ages are given with an error of $1 \sigma$, including the instrumental error. Evaluation of the error is based on the scattering of separate measurements of the count rate of the sample, standard, and background. This method modifies that suggested by Grevecoeur et al (1959).

The mineral composition of shells and corals was determined with the aid of a DRON-1 X-ray diffractometer. The standard used for calcite was Icelandic spar and for aragonite modern coral. To make qualitative determination of mineral composition each sample was analyzed in the range of $14-40^{\circ} 2 Q^{\prime \prime}$. Quantitative determination of calcite and aragonite in the sample was carried out by the internal standard procedure (Gorbunova, 1969).

When the sample is originally composed of aragonite, X-ray diffractometric analysis determines the degree of recrystallization to calcite. For such samples, ages are quoted with the results of the X-ray analysis, as recryst $=-\%$.

Although the dates were not corrected for recrystallization, we can identify four age groups according to the effect of recrystallization upon radiocarbon age: Group 1 - samples 500 to 5000 years - the error at maximum recrystallization, $90 \%$, does not exceed 1000 years; Group 2 samples 5 to 20,000 years - the error amounts to several thousands years; Group 3 - samples 20 to 35,000 years - the error averages 5 to 20,000 years; Group 4 - samples $>35,000$ years - in this group, even an insignificant degree of recrystallization can cause an error of 20,000 years or more which means that these ancient samples are not suitable for age determination. 
In addition to the conventional instruments, an amplitude-differential discriminator (ADD) designed at the Laboratory was used in the electronics. Up to five radiocarbon counters can be connected to this discriminator allowing simultaneous measurement of several samples (Khait \& Shlukov, 1973). This was achieved by employing the principle of signal compression applied in communication.

\section{ACKNOWLEDGMENTS}

The authors fully acknowledge the valuable guidance, advice, and the selection of samples by the Head of the Laboratory, P A Kaplin. We also express our special recognition to M G Groswald, Institute of Geography, Academy of Sciences of the USSR, and to Yu A Pavlidis, Institute of Oceanology, Academy of Sciences of the USSR, for the unique collection of samples placed at our disposal. The authors also thank N I Sarazhinskaya for preparation of samples.

\section{Pacific Islands $I$}

Samples coll from Pacific Is during 6th cruise of $R_{/}^{\prime} V$ Dmitry Mendeleyey and subm by $\mathbf{P}$ A Kaplin, MGU.

\section{Efate Island series}

MGU-174. Efate I.

$$
\mathbf{2 8 9 0} \pm \mathbf{5 0 0}
$$

recryst $=10 \%$

Coral limestone from Efate I., New Hebrides, S coast of island, W coast of Eracor bay $\left(17^{\circ} 46^{\prime} 24^{\prime \prime} \mathrm{S}, 166^{\circ} 16^{\prime} 39^{\prime \prime} \mathrm{E}\right)$. Scarp of 1st terrace, alt $1.5 \mathrm{~m}$.

\section{MGU-175. Efate I.}

$22,400 \pm 100$

Coral limestone, $\mathrm{S}$ coast of island, Pango peninsula, Etumoo plateau $\left(17^{\circ} 46^{\prime} 00^{\prime \prime} \mathrm{S}, 168^{\circ} 16^{\prime} 47^{\prime \prime} \mathrm{E}\right)$. Scarp of Pleistocene upper terrace, alt $20 \mathrm{~m}$, with well expressed wave-cut recess at foot. Absolute elev of sampling, $70 \mathrm{~m}$.

\section{MGU-170-1. Efate I.}

$3960 \pm 150$

NW coast of island, Samoa cape $\left(17^{\circ} 34^{\prime} 12^{\prime \prime} \mathrm{S}, 168^{\circ} 14^{\prime} 59^{\prime \prime} \mathrm{E}\right)$. Scarp of 1 st terrace, alt $2 \mathrm{~m}$. Terrace, elev Holocene coastal accumulative form composed of coral fragments, shell detritus, and pieces of coral limestone. This and 2 subsequent samples date ancient accumulative form. Present elev reaches $+8 \mathrm{~m}$.

MGU-170.2. Efate I.

$3740 \pm 250$ recryst $=5 \%$

Sample from same scarp of ancient accumulative form as MGU-170$1\left(17^{\circ} 34^{\prime} 12^{\prime \prime} \mathrm{S}, 168^{\circ} 14^{\prime} 59^{\prime \prime} \mathrm{E}\right)$. Material of filler, $i e$, calcareous sand, silt, and ooze.

MGU-170-3. Efate I.

Fragment of Tridacna shell from same scarp as MGU-170-1 (17० $34^{\prime}$ $\left.12^{\prime \prime} \mathrm{S}, 168^{\circ} 14^{\prime} 59^{\prime \prime} \mathrm{E}\right)$. 
MGU-171. Efate I.

Coral limestone from NE coast of island $\left(17^{\circ} 34^{\prime} 36^{\prime \prime} \mathrm{S}, 168^{\circ} 28^{\prime} 58^{\prime \prime}\right.$ E). Reef terraces, $+1.5,+15$ and $+29 \mathrm{~m}$, composed of coral limestone, well-expressed on coast. Barometric profile was run through terraces. Dates 1st terrace, $+1.5 \mathrm{~m}$.

MGU-172. Efate I.

$15,220 \pm 300$ recryst $=90 \%$

Coral limestone from NE coast of island $\left(17^{\circ} 34^{\prime} 42^{\prime \prime} \mathrm{S}, 168^{\circ} 28^{\prime} 49^{\prime \prime}\right.$ E). Scarp of 2 nd terrace, $+15 \mathrm{~m}$. Same barometric profile as MGU-171. Dates 2nd terrace.

MGU-173. Efate I.

$\mathbf{2 9 , 8 8 0} \pm \mathbf{3 0 0}$ recryst $=90 \%$

Coral limestone from NE coast of island $\left(17^{\circ} 34^{\prime} 48^{\prime \prime} \mathrm{S}, 168^{\circ} 28^{\prime} 37^{\prime \prime}\right.$ E). Same barometric profile as MGU-171 and -172. Dates $3 \mathrm{~d}$ terrace, $+29 \mathrm{~m}$.

MGU-176. Efate I.

$21,400 \pm 270$

Coral limestone from $\mathrm{S}$ coast of island (17 $48^{\prime} 36^{\prime \prime} \mathrm{S}, 168^{\circ} 22^{\prime} 42^{\prime \prime}$ E), de la Taome bay. Foot of ancient cliff and adjacent elev Holocene accumulative coastal form, $+14 \mathrm{~m}, 130 \mathrm{~m}$ from shore line.

MGU-177. Efate I.

$2500 \pm 190$

recryst $=10 \%$

Coral limestone, $\mathrm{S}$ coast of island, Traqoi bay $\left(17^{\circ} 49^{\prime} 24^{\prime \prime} \mathrm{S}, 168^{\circ}\right.$ $\left.24^{\prime} 45^{\prime \prime} \mathrm{E}\right)$. Reef flat, tide level.

MGU-178. Efate I.

$1666 \pm 70$

Coral limestone, S coast of island $\left(17^{\circ} 49^{\prime} 24^{\prime \prime} \mathrm{S}, 168^{\circ} 24^{\prime} 45^{\prime \prime} \mathrm{E}\right)$. Surface of 1 st terrace, $+1.5 \mathrm{~m}$, ie, tide level.

\section{New Guinea series}

MGU-162. New Guinea I.

$21,920 \pm 360$

Coral limestone, NE coast, SW of Madang, middle course of Gogol $\mathrm{R}\left(5^{\circ} 17^{\prime} 00^{\prime \prime} \mathrm{S}, 145^{\circ} 38^{\prime} 00^{\prime \prime} \mathrm{E}\right)$. Hillside, left-hand bank of river. Height of sampling $+80 \mathrm{~m}$.

MGU-163. New Guinea I.

$29,780 \pm 1000$

Coral limestone, NE coast of island, SW of Madang, middle course of Gogol R (5 $\left.17^{\prime} 00^{\prime \prime} \mathrm{S}, 145^{\circ} 38^{\prime} 00^{\prime \prime} \mathrm{E}\right)$. Same hillside as MGU-162 but $20 \mathrm{~m}$ higher. Absolute elev, $+100 \mathrm{~m}$.

MGU-164. New Guinea I.

Coral fragment, NE coast of island, $\mathrm{S}$ of Madang, middle course of Gogol R $\left(5^{\circ} 17^{\prime} 30^{\prime \prime} \mathrm{S}, 145^{\circ} 40^{\prime} 00^{\prime \prime} \mathrm{E}\right)$. Sample taken from road quarry of dark-blue clay enclosing debris of shells and corals. Alt, ca $+50 \mathrm{~m}$. 
MGU-165. New Guinea I.

$\mathbf{3 1 , 6 0 0} \pm \mathbf{5 0 0}$

Fossil wood, NE coast of island, S of Madang $\left(5^{\circ} 17^{\prime} 00^{\prime \prime} \mathrm{S}, 145^{\circ} 41^{\prime}\right.$ $00^{\prime \prime} \mathrm{E}$ ). Road quarry, dark-blue clay base containing calcite concretions, shell fragments, and fossil wood. Alt, ca $+60 \mathrm{~m}$.

\section{MGU-181. New Guinea I.}

$$
\mathbf{2 5 , 1 0 0} \pm \mathbf{7 8 0}
$$

Coral fragments, NE coast of island, SW of Madang $\left(5^{\circ} 17^{\prime} 00^{\prime \prime} \mathrm{S}\right.$, $\left.145^{\circ} 42^{\prime} 00^{\prime \prime} \mathrm{E}\right)$. Same road quarry as MGU-165, bed of coral fragments and filler overlying dark-blue clay. Coral fragments are angular with well-preserved structure.

\section{Funafuti Atoll series}

MGU-185. Funafuti Atoll

Lithified coral, N part of atoll rim, Amatuku I. $\left(8^{\circ} 26^{\prime} 12^{\prime \prime} \mathrm{S}, 179^{\circ}\right.$ $\left.11^{\prime} 07^{\prime \prime} \mathrm{E}\right)$. Surface of reef flat, ocean.ward side, tide flooded.

MGU-186. Funafuti Atoll

$1725 \pm 120$

Coral fragment, $\mathrm{N}$ part of atoll rim, Amatuku I. $\left(8^{\circ} 26^{\prime} 18^{\prime \prime} \mathrm{S}, 179^{\circ}\right.$ $\left.11^{\prime} 12^{\prime \prime} \mathrm{E}\right)$. Lagoon side, washout scarp revealing accumulative body of island. Elev, $+0.5 \mathrm{~m}$.

MGU-190. Funafuti Atoll $\mathbf{8 3 0} \pm \mathbf{5 0}$

Coral, SE part of atoll rim, Funafara I. ( $\left.8^{\circ} 31^{\prime} 57^{\prime \prime} \mathrm{S}, 179^{\circ} 06^{\prime} 10^{\prime \prime} \mathrm{E}\right)$. Reef flat, lagoon side, 5 to $10 \mathrm{~m}$ above tide level. Coral Porites in growth position.

MGU-191. Funafuti Atoll

$1823 \pm 100$

Coral, SW part of atoll rim, Funafara I. $\left(8^{\circ} 30^{\prime} 33^{\prime \prime} \mathrm{S}, 179^{\circ} 02^{\prime} 15^{\prime \prime}\right.$

E). Rim flooded by tide, coral Porites in growth position.

MGU-235. Funafuti Atoll

Recent

Sediments of Halimeda calcareous algae, base of atoll lagoon $\left(8^{\circ} 31^{\prime}\right.$ $57^{\prime \prime} \mathrm{S}, 179^{\circ} 08^{\prime} 12^{\prime \prime} \mathrm{E}$ ). Water depth, $40 \mathrm{~m}$. Piston core, 0 to $10 \mathrm{~cm}$ sub-base.

MGU-236. Funafuti Atoll

$440 \pm 90$

Sediments of Halimeda calcareous algae, bottom of atoll's lagoon $\left(8^{\circ} 31^{\prime} 57^{\prime \prime} \mathrm{S}, 179^{\circ} 08^{\prime} 12^{\prime \prime} \mathrm{E}\right)$. Sample from same piston core as MGU235, interval 196 to $202 \mathrm{~cm}$ sub-base.

MGU-237. Funafuti Atoll $530 \pm 60$

Sediments of Halimeda calcareous algae, base of atoll lagoon $\left(8^{\circ} 31^{\prime}\right.$ $\left.57^{\prime \prime} \mathrm{S}, 179^{\circ} 08^{\prime} 12^{\prime \prime} \mathrm{E}\right)$. Sample from same piston core as MGU-235, interval 340 to $350 \mathrm{~cm}$ sub-base.

\section{Butaritari Atoll series}

MGU-238. Butaritari Atoll

Recent

Coral sand, base of atoll lagoon ( $\left.3^{\circ} 09^{\prime} 00^{\prime \prime} \mathrm{S}, 172^{\circ} 51^{\prime} 36^{\prime \prime} \mathrm{E}\right)$. Water depth, $33 \mathrm{~m}$. Piston core, interval 0 to $10 \mathrm{~cm}$ sub-base. 
MGU-239. Butaritari Atoll

Coral sand with coral and shell detritus, base of atoll lagoon $\left(3^{\circ} 09^{\prime}\right.$ $00^{\prime \prime} \mathrm{S}, 172^{\circ} 51^{\prime} 36^{\prime \prime} \mathrm{E}$ ). Sample taken from same piston core as MGU-238, interval 98 to $104 \mathrm{~cm}$ sub-base.

\section{Lord-How Island series}

MGU-180. Lord-How I. Beach.

Shell fragments, W coast of island $\left(31^{\circ} 32^{\prime} 20^{\prime \prime} \mathrm{S}, 159^{\circ} 04^{\prime} 50^{\prime \prime} \mathrm{E}\right)$.

MGU-179. Lord-How I.

$20,000 \pm 220$

Calcarenite (lithified eolian coral sand), W coast of island, between Signal cape and Lover bay ( $\left.31^{\circ} 32^{\prime} 00^{\prime \prime} \mathrm{S}, 159^{\circ} 04^{\prime} 30^{\prime \prime} \mathrm{E}\right)$. Outlies ancient dune over coastal aggradation terrace. Elev, $2 \mathrm{~m}$ above mean water level.

\section{MGU-187. Lord-How I.}

$$
\mathbf{2 5 , 1 6 0} \pm \mathbf{5 0 0}
$$

recryst $=60 \%$

Slightly lithified carbonate sand (eolian horizon), NE coast of island, Ned's Beach bay $\left(31^{\circ} 31^{\prime} 03^{\prime \prime} \mathrm{S}, 159^{\circ} 04^{\prime} 36^{\prime \prime} \mathrm{E}\right)$. Cliff in ancient dune, 2nd layer from top. Elev, $+24 \mathrm{~m}$.

MGU-184-1. Lord-How I.

Coral fragments, NE coast of island, Ned's Beach bay $\left(31^{\circ} 31^{\prime} 03^{\prime \prime} \mathrm{S}\right.$, $\left.159^{\circ} 04^{\prime} 36^{\prime \prime} \mathrm{E}\right)$. Sample taken from foot of same cliff as MGU-187. Elev, $+3.5 \mathrm{~m}$. Sand with shell detritus, coral fragments, and gravels of volcanic rocks (coastal-marine deposits). Dates period of high sea level.

MGU-184-2. Lord-How I.

$29,590 \pm 600$

Coral sand and detritus, NE coast, Ned's Beach bay $\left(31^{\circ} 31^{\prime} 03^{\prime \prime} \mathrm{S}\right.$, $159^{\circ} 04^{\prime} 36^{\prime \prime} \mathrm{E}$ ). Sample taken from same cliff as MGU-184-1.

\section{Pacific Islands II}

Samples coll from Pacific Is during 6th Cruise of $R / V$ Kallisto.

\section{MGU-629. Bagaman I.}

$$
\begin{array}{r}
\mathbf{3 1 9 0} \pm \mathbf{1 7 0} \\
\text { recryst }=5 \%
\end{array}
$$

Beach-rock (cemented Tridacna shell) from Bagaman I., Calvados Is, $S$ coast, Lalagela bay $\left(11^{\circ} 08^{\prime} 35^{\prime \prime} \mathrm{S}, 152^{\circ} 40^{\prime} 51^{\prime \prime} \mathrm{E}\right)$. Rear part of beach, ancient storm accumulation bar. Elev, $20 \mathrm{~cm}$ above tide level.

MGU-593. Misima I.

$$
22,500 \pm 180
$$
recryst $=70 \%$

Coral fragments from Misima I, Luisiade archipelago, Bvaga bay $\left(10^{\circ} 41^{\prime} 12^{\prime \prime} \mathrm{S}, 152^{\circ} 50^{\prime} 47^{\prime \prime} \mathrm{E}\right)$. Surface of terrace, $5 \mathrm{~m}$ alt.

MGU-633. Misima I.

$12,316 \pm 210$

recryst $=100 \%$

Lithified coral fragments, Bvaga bay $\left(10^{\circ} 41^{\prime} 12^{\prime \prime} \mathrm{S}, 152^{\circ} 50^{\prime} 47^{\prime \prime} \mathrm{E}\right)$. Scarp of terrace, $5 \mathrm{~m}$ alt. Elev, $+1.5 \mathrm{~m}$. 


\section{Bio Island series}

MGU-634. Bio I.

$31,930 \pm 1300$

recryst $=95 \%$

Reef limestone from Bio I., Solomon Is $\left(10^{\circ} 10^{\prime} 06^{\prime \prime} \mathrm{S}, 161^{\circ} 41^{\prime} 00^{\prime \prime}\right.$ E). Surface of $3 \mathrm{~d}$ terrace (highest elev on island). Elev, $+34 \mathrm{~m}$. Limestone overlain by $50 \mathrm{~cm}$ soil.

MGU-627. Bio I.

Reef limestone from NNW side of island $\left(10^{\circ} 10^{\prime} 03^{\prime \prime} \mathrm{S}, 161^{\circ} 41^{\prime} 30^{\prime \prime}\right.$ E). Reef flat, high tide level.

MGU-624. Bio I.

$\mathbf{2 4 6 7} \pm \mathbf{8 4}$ recryst $=70 \%$

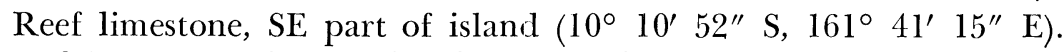
Scarp of 1 st terrace, $+2.5 \mathrm{~m}$ alt. Elev, $0.8 \mathrm{~m}$ above high tide level.

MGU-591. Bio I.

$3180 \pm 170$

Reef limestone, SE part of island $\left(10^{\circ} 10^{\prime} 52^{\prime \prime} \mathrm{S}, 161^{\circ} 41^{\prime} 15^{\prime \prime} \mathrm{E}\right)$. Scarp of 1 st terrace same as MGU-624. Elev, $0.3 \mathrm{~m}$ above high tide level.

\section{MGU-631. Bio I.}

$\mathbf{2 3 , 8 9 4} \pm \mathbf{4 3 0}$ recryst $=100 \%$

Reef limestone, SW part of island $\left(10^{\circ} 10^{\prime} 17^{\prime \prime} \mathrm{S}, 161^{\circ} 40^{\prime} 40^{\prime \prime} \mathrm{E}\right)$. Scarp of 2nd terrace, $3.5 \mathrm{~m}$ alt, with wave-cut recess at foot, very steep, descending to 1 st terrace, $2.5 \mathrm{~m}$ alt. Elev, $1.3 \mathrm{~m}$ above 1 st terrace.

MGU-592. Bio I.

$\mathbf{2 6 , 8 7 0} \pm \mathbf{3 5 0}$ recryst $=80 \%$

Reef limestone, SW part of island $\left(10^{\circ} 10^{\prime} 17^{\prime \prime} \mathrm{S}, 161^{\circ} 40^{\prime} 40^{\prime \prime} \mathrm{E}\right)$. Scarp of 2nd terrace same as MGU-631. Elev, $166 \mathrm{~cm}$ above 1st terrace.

MGU-618. Bio I.

$$
4100 \pm 84
$$

recryst $=45 \%$

Reef limestone, SW part of island $\left(10^{\circ} 10^{\prime} 17^{\prime \prime} \mathrm{S}, 161^{\circ} 40^{\prime} 40^{\prime \prime} \mathrm{E}\right)$. Surface of 1st terrace, rear part. Depth, $55 \mathrm{~cm}$ under soil.

MGU-630. Norfolk I.

$$
\begin{array}{r}
\mathbf{6 4 7 8} \pm \mathbf{1 1 6} \\
\text { recryst }=60 \%
\end{array}
$$

Lithified sand (calcarenite), SE part of island (29 $09^{\prime} 30^{\prime \prime} \mathrm{S}, 167^{\circ}$ $57^{\prime} 50^{\prime \prime} \mathrm{E}$ ). Contact between Hunter cape accumulative terrace and shore. Sample taken from base of layer $185 \mathrm{~cm}$ thick.

MGU-628. Norfolk I.

Lithified sand (calcarenite), S part of island, Hunter cape $\left(29^{\circ} 03^{\prime}\right.$ $\left.45^{\prime \prime} \mathrm{S}, 167^{\circ} 57^{\prime} 30^{\prime \prime} \mathrm{E}\right)$. Ancient dune, $16 \mathrm{~m}$ alt. Sample taken in cliff at $1 \mathrm{~m}$ above high tide level. 
Niuye Island series

MGU-589. Niuye I.

recryst $=90 \%$

Reef limestone, SE coast $\left(19^{\circ} 06^{\prime} 43^{\prime \prime} \mathrm{S}, 169^{\circ} 48^{\prime} 54^{\prime \prime} \mathrm{E}\right)$. Reef flat, exposed only at low tide.

MGU-590. Niuye I.

$19,580 \pm 460$ recryst $=10 \%$

Reef limestone, SE coast $\left(19^{\circ} 06^{\prime} 43^{\prime \prime} \mathrm{S}, 169^{\circ} 48^{\prime} 54^{\prime \prime} \mathrm{E}\right)$. Surface of terrane $3 \mathrm{~m}$ above low tide level.

MGU-622. Niuye I.

$27,480 \pm 330$

Reef limestone, SE coast $\left(19^{\circ} 06^{\prime} 43^{\prime \prime} \mathrm{S}, 169^{\circ} 48^{\prime} 54^{\prime \prime} \mathrm{E}\right)$. Scarp of terrace near brow. Elev, $20 \mathrm{~m}$.

\section{MGU-625. Niuye I.}

$28,968 \pm 1020$

recryst $=75 \%$

Reef limestone, SE coast $\left(19^{\circ} 06^{\prime} 43^{\prime \prime} \mathrm{S}, 169^{\circ} 48^{\prime} 54^{\prime \prime} \mathrm{E}\right)$. Top of ancient rim of elev atoll. Elev, $65 \mathrm{~m}$.

MGU.632. Suvorov Atoll

$3623 \pm 116$

recryst $=30 \%$

Coral fragments, W part of atoll, High I. (13 $16^{\prime} 10^{\prime \prime} \mathrm{S}, 163^{\circ} 11^{\prime} 42^{\prime \prime}$ E). Reef flat, oceanward side, flooded by tide in middle.

MGU-626. Suvorov Atoll

$1197 \pm 170$

Lithified coral (reef-rock), W part of atoll, High I. $\left(13^{\circ} 16^{\prime} 10^{\prime \prime} \mathrm{S}\right.$, $\left.163^{\circ} 11^{\prime} 35^{\prime \prime} \mathrm{E}\right)$. Central part covered by soil, $15 \mathrm{~cm}$ thick. Elev, $2.5 \mathrm{~m}$.

MGU-623. Puka-Puka Atoll

Recent

Cemented beach sand (beach-rock), SE part of atoll, Motu Ko I., oceanward side $\left(10^{\circ} 57^{\prime} 00^{\prime \prime} \mathrm{S}, 165^{\circ} 47^{\prime} 35^{\prime \prime} \mathrm{E}\right)$. Contact between reef flat and foot of pebble bar. Elev, $0.5 \mathrm{~m}$ above low tide level.

\section{Cuba}

Samples subm by Yu A Pavlidis, Inst Oceanol, Acad Sci USSR.

\section{Island of Pines series}

MGU-363. I. of Pines

Mollusk shells, S coast of island (21 $\left.32^{\prime} 56^{\prime \prime} \mathrm{N}, 82^{\circ} 45^{\prime} 24^{\prime \prime} \mathrm{W}\right)$. 2nd terrace, quarry. Depth, 3 to $4 \mathrm{~m}$ from ground surface.

\section{MGU-465. I. of Pines}

Roots of mangrove trees, SW coast of island, Colony region $\left(21^{\circ} 38^{\prime}\right.$ $\left.54^{\prime \prime} \mathrm{N}, 82^{\circ} 58^{\prime} 18^{\prime \prime} \mathrm{W}\right)$. Base of ancient accumulative form. Depth, $1.4 \mathrm{~m}$ from ground surface. 
Organic ooze, Siguance bay $\left(24^{\circ} 43^{\prime} 00^{\prime \prime}\right.$ N, $83^{\circ} 05^{\prime} 00^{\prime \prime}$ W). Water depth of $10 \mathrm{~m}$. Depth, 340 to $380 \mathrm{~cm}$ sub-base.

MGU-368. I. of Pines $\quad \mathbf{7 3 6 0} \pm \mathbf{2 9 0}$

Sapropel, water depth, $10 \mathrm{~m}\left(21^{\circ} 43^{\prime} 00^{\prime \prime} \mathrm{N}, 83^{\circ} 05^{\prime} 00^{\prime \prime}\right.$ W). Iepth 380 to $435 \mathrm{~cm}$ sub-base.

MGU-369. I. of Pines

$4055 \pm 120$

Peat, Siguance bay $\left(21^{\circ} 43^{\prime} 48^{\prime \prime}\right.$ N, $83^{\circ} 03^{\prime} 12^{\prime \prime}$ W). Water depth, $3 \mathrm{~m}$. Depth, 10 to $45 \mathrm{~cm}$ sub-base.

MGU-414. I. of Pines

$1610 \pm 130$

Organic limestone, $\mathrm{S}$ coast of island, Playa Larga $\left(21^{\circ} 28^{\prime} 45^{\prime \prime} \mathrm{N}\right.$, $\left.82^{\circ} 44^{\prime} 45^{\prime \prime} \mathrm{W}\right)$. 2nd marine terrace.

\section{MGU-415. I. of Pines}

$16,430 \pm 200$ recryst $=70 \%$

Oolitic sandstone, $S$ coast of island $\left(21^{\circ} 28^{\prime} 45^{\prime \prime} \mathrm{N}, 82^{\circ} 44^{\prime} 45^{\prime \prime} \mathrm{W}\right)$. 2nd marine terrace. Depth, 6 to $7 \mathrm{~m}$ below ground surface.

MGU.416. I. of Pines

$\mathbf{2 0 , 0 0 0} \pm \mathbf{2 6 0}$ recryst $=10 \%$

Coral, $\mathrm{S}$ coast of island $\left(21^{\circ} 28^{\prime} 45^{\prime \prime} \mathrm{W}, 82^{\circ} 44^{\prime} 45^{\prime \prime} \mathrm{W}\right)$. 2nd marine terrace.

\section{Island of Cuba series}

MGU.371. I. of Cuba

Peat, Inacos bar, Molase cape $\left(23^{\circ} 12^{\prime} 28^{\prime \prime} \mathrm{N}, 81^{\circ} 07^{\prime} 30^{\prime \prime} \mathrm{W}\right)$. Water depth, $2 \mathrm{~m}$. Depth, 20 to $30 \mathrm{~cm}$ sub-base.

\section{MGU-417. I. of Cuba

Coral, N coast of island, Guanos cape (23 $\left.09^{\prime} 12^{\prime \prime} \mathrm{N}, 81^{\circ} 39^{\prime} 15^{\prime \prime} \mathrm{W}\right)$. 1st marine terrace. Depth of sampling, $1 \mathrm{~m}$ below ground surface.

MGU-418. I. of Cubar $1170 \pm 130$

Coral, N coast of island, Guanos cape $\left(23^{\circ} 09^{\prime} 12^{\prime \prime} \mathrm{N}, 81^{\circ} 39^{\prime} 15^{\prime \prime} \mathrm{W}\right)$. 1 st marine terrace. Depth of sanmpling, $1 \mathrm{~m}$ below ground surface.

MGU-419. I. of Cuba

$1900 \pm 120$

Shell, N coast of island, Guanos cape (23० $\left.09^{\prime} 12^{\prime \prime} \mathrm{N}, 81^{\circ} 39^{\prime} 15^{\prime \prime} \mathrm{W}\right)$.

lst marine terrace. Depth, $1 \mathrm{~m}$ below ground surface.

MGU-547. I. of Cuba $\quad 15,020 \pm 300$

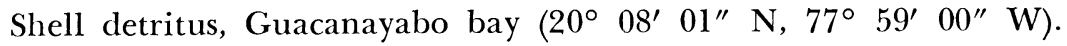
Shelf, water depth, $26 \mathrm{~m}$. Depth, 310 to $330 \mathrm{~cm}$ sub-base.

MGU-548. I. of Cuba

$$
\mathbf{7 4 8 0} \pm \mathbf{8 0 0}
$$

recryst $=10 \%$

Shell, Guacanayabo bay $\left(20^{\circ} 21^{\prime} 30^{\prime \prime} \mathrm{N}, 77^{\circ} 24^{\prime} 30^{\prime \prime} \mathrm{W}\right)$. Shelf, water depth, $9 \mathrm{~m}$. Depth, 420 to $440 \mathrm{~cm}$ sub-base. 
MGU-550. I. of Cuba

Shell detritus, NE coast of island, Nine bay $\left(20^{\circ} 48^{\prime} 00^{\prime \prime} \mathrm{N}, 75^{\circ} 37^{\prime}\right.$ $\left.30^{\prime \prime} \mathrm{W}\right)$. Water depth, $24 \mathrm{~m}$. Depth, 340 to $360 \mathrm{~cm}$ sub-base.

MGU-551. I. of Cuba

$7680 \pm 150$

recryst $=10 \%$

Shell detritus, Guacanayabo bay $\left(20^{\circ} 21^{\prime} 30^{\prime \prime} \mathrm{N}, 77^{\circ} 24^{\prime} 30^{\prime \prime} \mathrm{W}\right)$. Shelf, water depth, $22 \mathrm{~m}$. Depth, 275 to $295 \mathrm{~cm}$ sub-base.

MGU-552. I. of Cuba

$3370 \pm 80$

Shell detritus, Onda bay $\left(22^{\circ} 56^{\prime} 11^{\prime \prime} \mathrm{N}, 83^{\circ} 10^{\prime} 50^{\prime \prime} \mathrm{W}\right)$. Water depth, $4 \mathrm{~m}$. Depth, 400 to $455 \mathrm{~cm}$ sub-base.

\section{Canada}

Samples subm by M G Groswald, Inst Geog, Acad Sci USSR.

\section{Queen Elizabeth Islands series}

MGU-331. Queen Elizabeth Is

$8570 \pm 120$

Mollusk shell, Devon I., Greenell peninsula $\left(76^{\circ} 23^{\prime} 00^{\prime \prime}\right.$ N, $95^{\circ} 31^{\prime}$ $\left.00^{\prime \prime} \mathrm{W}\right)$. Ancient shore line. Alt, $+98 \mathrm{~m}$.

\section{MGU-330. Queen Elizabeth Is}

$11,280 \pm 160$

Mollusk shell, Devon I., Greenell peninsula $\left(76^{\circ} 15^{\prime} 00^{\prime \prime} \mathrm{N}, 95^{\circ} 16^{\prime}\right.$ $\left.00^{\prime \prime} \mathrm{W}\right)$. Ancient shore line. Alt, $+84 \mathrm{~m}$.

MGU-332. Queen Elizabeth Is

Mollusk shell, Devon I., SW coast of Greenell peninsula ( $76^{\circ} 23^{\prime} 00^{\prime \prime}$ $\left.\mathrm{N}, 95^{\circ} 37^{\prime} 00^{\prime \prime} \mathrm{W}\right)$. Ancient shore line. Alt, +44.5m.

\section{MGU.333. Queen Elizabeth Is}

Mollusk shell, Devon I., SW coast of Greenell peninsula $\left(76^{\circ} 23^{\prime} 00^{\prime \prime}\right.$ $\left.\mathrm{N}, 95^{\circ} 37^{\prime} 00^{\prime \prime} \mathrm{W}\right)$. Ancient shore line. Alt, $+62 \mathrm{~m}$.

\section{MGU-334. Queen Elizabeth Is}

Mollusk shell, Devon I., SW coast of Greenell peninsula ( $76^{\circ} 23^{\prime} 00^{\prime \prime}$ $\left.\mathrm{N}, 95^{\circ} 37^{\prime} 00^{\prime \prime} \mathrm{W}\right)$. Ancient shore line. Alt, $+150 \mathrm{~m}$.

\section{REFERENCES}

Gorbunova, Z N, 1969, X-ray diffraction method of determination of carbonates, quartz and other minerals in sediments: Litologiya i Poleznye Iskopayemye (Lithology \& Econ Minerals), no. 2, p 75-80.

Grevecoeur, E H, Stricht, A V, and Capron, P S, 1959, Precision of the dating method. Standardization of the calculation of the errors and of the maximum age in the ${ }^{14} \mathrm{C}$ method: Acad Royal Bergique Bull Cl: Science, v 45, p 876-890.

Khait, V Z and Shlukov, A I, 1973, Use of one amplitude-differential discriminator for simultaneous measurement of radioactivity in several samples: Pribory i Tekhnika Eksperimenta (Experimental Instruments I Techniques), no. 6, p 60-61. 